\title{
The Fabrication of the Microfluidic Device with Micro- and Nano- Scale Channels
}

\author{
Guangchun Fu \\ Department of Mechanical and Electrical Engineering, \\ Xiamen University, China \\ Pen-Tung Sah Institute of Micro-Nano Science and \\ Technology, \\ Xiamen University, China
}

Yang Li

Department of Materials Science \& Engineering, Xiamen University, China

Pen-Tung Sah Institute of Micro-Nano Science and Technology,

Xiamen University, China

\author{
Zezhi Zheng \\ Department of Physics, Xiamen University, China \\ Pen-Tung Sah Institute of Micro-Nano Science and \\ Technology, \\ Xiamen University, China
}

\author{
Hong Chen \\ Pen-Tung Sah Institute of Micro-Nano Science and \\ Technology, \\ Xiamen University, China
}

\begin{abstract}
Here a simple method for fabrication of the microfluidic device with micro- and nanochannels was developed. The microchannels in micro and nano scales were fabricated on a glass substrate during a two- step process respectively. And then the glass substrate with the microchannels was bonded with another flat glass substrate at room temperature to avoid the collapse of the nanochannels. Standard UV lithography and wet etching were used in this method. The nanochannels with a series of depths were obtained with optimized etchant concentration of $1.5 \times 10^{-2} \mathrm{~mol} \mathrm{~L}^{-1} \mathrm{HF}, 7.5 \times 10^{-3} \mathrm{~mol} \mathrm{~L}^{-1} \mathrm{HNO}_{3}$ and $7.5 \times 10^{-3} \mathrm{~mol} \mathrm{~L}^{-1}$ $\mathrm{NH}_{4} \mathrm{~F}$, and the depth had a linear relationship with the etching time (the square of correlation coefficient was 0.9983 ). The roughness length of the etched nanochannel was $2.045 \mathrm{~nm}$ investigated by atomic force microscopy (AFM). The substrate was then bonded with a flat glass plate to form a closed microchannel, the cross-section of the bonded microchannel was imaged by a scanning electron microscopy (SEM). With this method, the microchannels combined with micro and nano scale can be prepared conveniently with low cost.
\end{abstract}

\section{Keywords-microfluidic device; nanochannel}

\section{INTRODUCTION}

Microfluidic devices have been proved a powerful tool and applied in many fields. With the shortened diffusion distance, the microfluidic device can realize fast and sensitive analysis with decreased consumption. When the dimension of the microchannel decreased into nanometer scale, the unique properties emerged such as overlapped electric double layer, increased surface tension, influence of the solid surface and quantum effect, which are very attractive for fundamental research. Because of the further decreased diffusion distance and confine space for nanoparticle and single molecule, the microfluidic device with nanochannel can be utilized in a wide range of applications, such as biomolecule analysis [1], detection [2].

The nanochannels were at least one of the dimensions smaller than $100 \mathrm{~nm}$ [3]. Many methods had been developed to fabricate the nanochannels; such as electron-beam lithography (EBL) [4], focused-ion beam (FIB) [5], proton beam writing (PBW) [6]. These methods realized the nanochannels with the precise control, but they were cost expensive and difficult to scale up. Surface nanomachining [7] was also used to fabricate the nanochannels by removing the sacrificial layer from the integrated device, but it also needed tens of hours of etching. Nanoimprint lithography [8] can only be used to fabricate the device with simply design. Buried channel technology [9] was very complex and chemical vapor deposition (CVD) was needed. All these methods did not satisfy the demand of the development of nanofluidic device. He [10] reported a method to fabricate the glass device by wet etching and room temperature bonding. The microchannels and nanochannels were etched in two glass substrates and then bonded together. During bonding, the two glass substrates needed to be aligned very precisely, and was not suitable for mass production. Here the microchannels and nanochannels were fabricated in the same glass substrate by two steps exposure, and then bonded to realized the final microfluidic device. By this method, the microfluidic device with microchannels and nanochannels can be prepared with simple, low cost and reliable characters.

\section{EXPERIMENT}

The fabrication of glass microfluidic device with the microchannels and nanochannels was according to the standard photolithographic and wet chemical etching protocol. A method with two steps was developed, one step for the fabrication of the microchannels, and the other for the nanochannels. The process was described briefly as the fig. 1 shown.

A SG2506 glass substrate with chromium and AZ1805 photoresist coating (Shaoguang Microelectronics Co., Changsha, China) was exposed through a photo mask by a MA6/BA6 mask aligner (SÜSS MicroTec AG, Garching, Germany). The photo mask was designed with the pattern of 
the microchannels, and the design was transferred onto the glass substrate. Then the microchannels were etched by $1 \mathrm{~mol}$ $\mathrm{L}^{-1} \mathrm{HF}$ and $1 \mathrm{~mol} \mathrm{~L}^{-1} \mathrm{NH}_{4} \mathrm{~F}$ at $40{ }^{\circ} \mathrm{C}$. The microchannels with the depth of $25 \mu \mathrm{m}$ were achieved after 20 min etching.

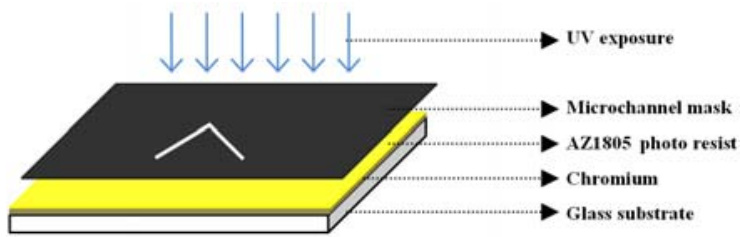

(a)

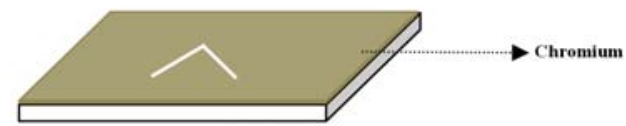

(b)

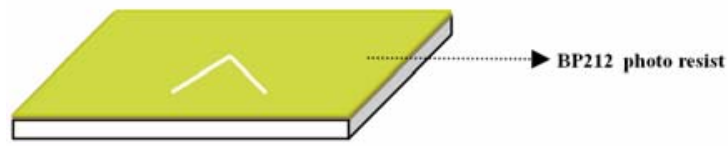

(c)

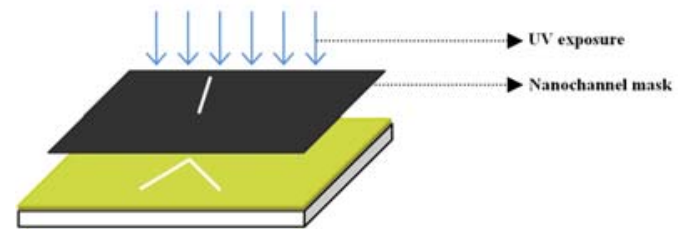

(d)

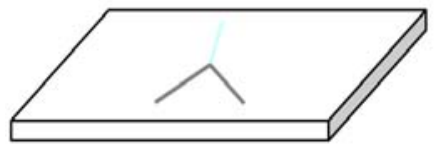

(e)

Fig. 1 Schematic diagram of the fabrication of the microfluidic device with microchannels and nanochannels.

The glass substrate with the microchannels was spun and coated by another photoresist (BP212), and a second UV exposure was carried out through another photo mask. On this photo mask, the pattern of the nanochannels was designed. Then the glass substrate was etched in diluted etchant $\left(1.5 \times 10^{-2} \mathrm{~mol} \mathrm{~L}^{-1} \mathrm{HF}, 7.5 \times 10^{-3} \mathrm{~mol} \mathrm{~L}^{-1} \mathrm{HNO}_{3}\right.$ and $7.5 \times 10^{-3}$ mol $\mathrm{L}^{-1} \mathrm{NH}_{4} \mathrm{~F}$ ) at $40{ }^{\circ} \mathrm{C}$. The depth of nanochannels was controlled by adjusting the etching time.

The access holes were drilled on the etched substrate at the ends of the microchannels and nanochannels. Then room temperature bonding was carried out to bond the etched substrate with a flat glass substrate, and then the closed channels were formed. The microfluidic chip was ready for use after aging for 3 7 days.

\section{RESULT AND DISCUSSION}

The etching of nanochannel was critical important for the whole process. Usually the microchannels were etched in the solution with high concentration, the etching rate was around $2.5 \mu \mathrm{m} \mathrm{min}^{-1}$. The etching time would be several seconds, and it was very difficult to realize the precise control over the nanochannel's depth. According to the report [10], the etchant with dilute concentration was adopted, and the etching time would increased into tens of minutes. As fig. 2 shown, the etching time was optimized and found to be linear with the channel's depth. The etching time was increased from $5 \mathrm{~min}$ to $30 \mathrm{~min}$, the nanochannel's depth also increased from 35.2 $\mathrm{nm}$ to $242.3 \mathrm{~nm}$. The square of correlation coefficient was 0.9983 , the etching rate was calculated to be $7.65 \mathrm{~nm} \mathrm{~min}^{-1}$. For each point in fig.2, the nanochannel' depth was measured by surface profiler at three different positions. The standard deviations in the fig. 2 proved that etching was very uniform for the whole glass substrate.

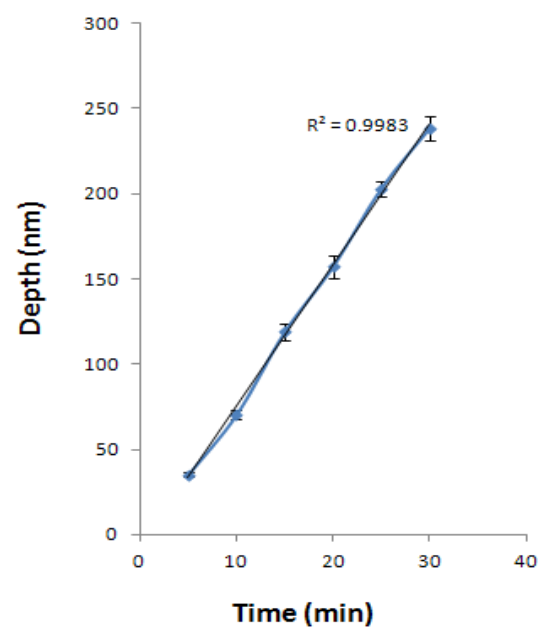

Fig. 2 The etching depth varied with the etching time. Etchant: $1.5 \times 10^{-2} \mathrm{~mol}$ $\mathrm{L}^{-1} \mathrm{HF}, 7.5 \times 10^{-3} \mathrm{~mol} \mathrm{~L}^{-1} \mathrm{HNO}_{3}$ and $7.5 \times 10^{-3} \mathrm{~mol} \mathrm{~L}^{-1} \mathrm{NH}_{4} \mathrm{~F}$. Etching temperature was $40^{\circ} \mathrm{C}$.

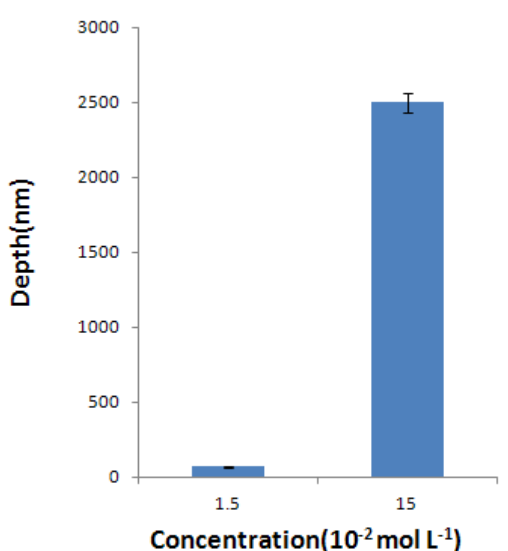

Fig. 3 The effect of etchant concentration on the depths of nanochannel. Molar concentration ratio of $\mathrm{HF}-\mathrm{HNO}_{3}-\mathrm{NH}_{4} \mathrm{~F}$ was 1:0.5:0.5, etching for 10 $\min$ at $40^{\circ} \mathrm{C}$.

The etchant's concentration had obvious influence on the channel's depth, and the etchant with two different 
concentrations was compared. The first etchant had $1.5 \times 10^{-2}$ mol L ${ }^{-1} \mathrm{HF}, 7.5 \times 10^{-3} \mathrm{~mol} \mathrm{~L}^{-1} \mathrm{HNO}_{3}$ and $7.5 \times 10^{-3} \mathrm{~mol} \mathrm{~L}^{-1}$ $\mathrm{NH}_{4} \mathrm{~F}$ (molar concentration ratio of $\mathrm{HF}: \mathrm{HNO}_{3}: \mathrm{NH}_{4} \mathrm{~F}=1$ : 0.5 : $0.5)$, the second etchant's concentration was ten times higher (the molar concentration ratio was kept the same). After 10 min's etching, the etching depth for the first etchant was $70.4 \pm 3.0 \mathrm{~nm}$, while the etching depth for the second one was $2497 \pm 66.5 \mathrm{~nm}$, which the result was shown in fig. 3 . The calculated etching rate was $250 \mathrm{~nm} \mathrm{~min}{ }^{-1}$, which was around 36 times more than the first etchant's.

Because high etching temperature would decrease the etchant's concentration for HF's volatility. $40{ }^{\circ} \mathrm{C}$ was chosen as the etching temperature to avoid the HF from volatilizing and keep the concentration constant.

By adjusting the etching time, the nanochannel with depths ranging from tens of nanometers to several hundreds nanometers can be realized. As shown in fig. 4, the average depth was $91.9 \mathrm{~nm}$ after $13 \mathrm{~min}$ etching. The roughness lengths before and after etching were $3.5 \mathrm{~nm}$ and $2.8 \mathrm{~nm}$ respectively. The etching did not increase the surface roughness of the glass substrate obviously. The depth of the nanochannel can also be confirmed by the SEM image of its cross section. In fig. 5, the microfluidic device was cut and scanned by SEM. The dark area in the middle was the nanochannel, whiles the gray areas above and below the nanochannel were the glass substrate. The depth of the nanochannel was $105 \mathrm{~nm}$. The top and bottom walls were also very smooth.

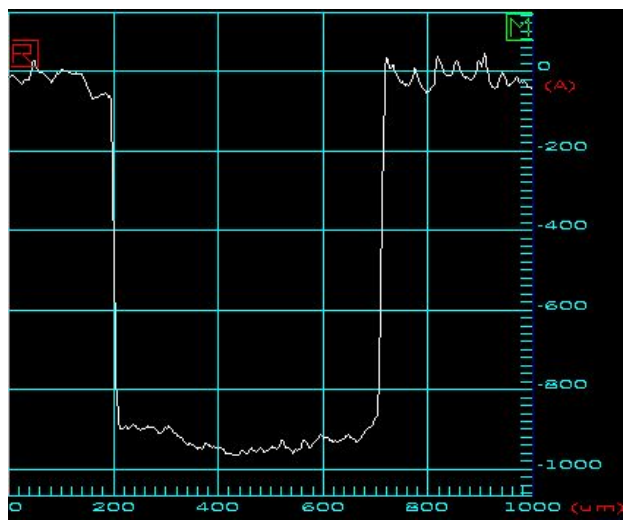

Fig. 4 The surface profile of the nanochannel. Etching for $13 \mathrm{~min}$, other conditions were the same as in Fig. 2.

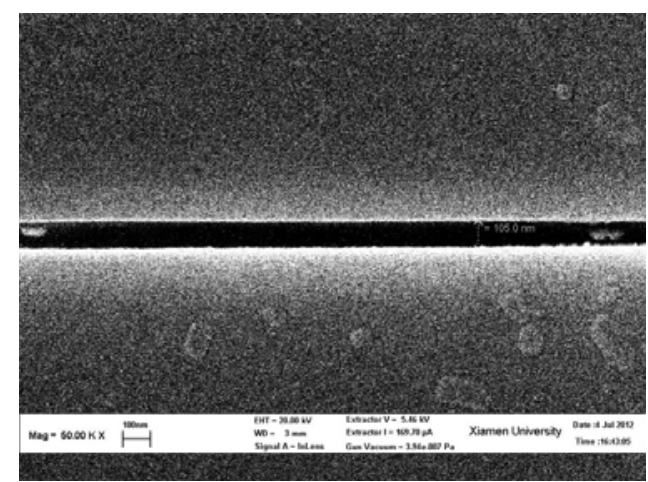

Fig. 5 The SEM image of the cross section of nanochannel.
Fig. 6 was the AFM image of the bottom and edge of nanochannel. The average roughness length of the etched nanochannel was $2.045 \mathrm{~nm}$, and the sharp peaks in fig 6a were the nanoparticle and nanofibers on the surface of nanochannel. The fig. 5b showed the edge of the nanochannel. The roughness of the edge was produced by the photo mask and photolithography.

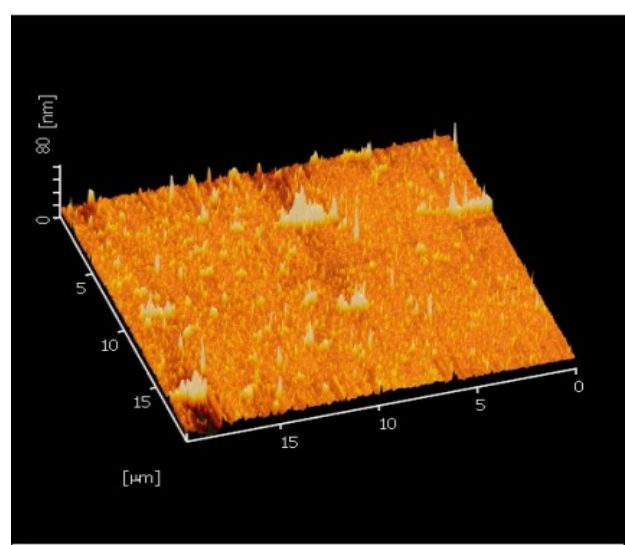

(a)

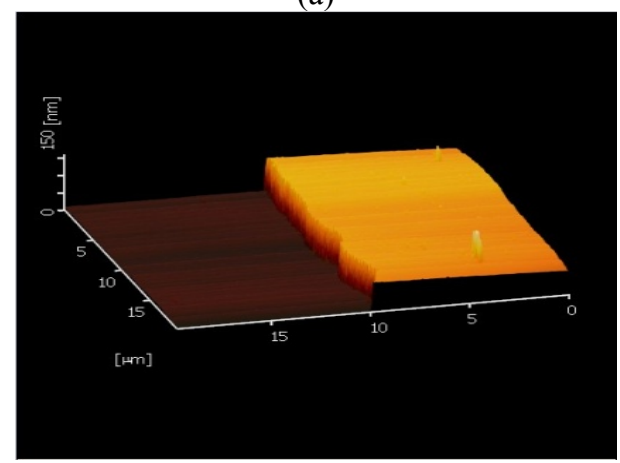

(b)

Fig. 6 The AFM images of nanochannel. (a) The roughness of nanochannel surface, $\mathrm{Ra}=2.045 \mathrm{~nm}$. (b) The 3D image of the edge of nanochannel, the average depth is $96 \mathrm{~nm}$.

\section{CONCLUSION}

Here we demonstrated a low cost and easy manipulated method for the fabrication of nanofluidic channels on glass substrates. With this method, a combined microchannels and nanochannels device was successfully fabricated. This technique can be used for the preparation of nanofluidic devices for the applications of chemical analysis, clinical diagnostic and many other purposes.

\section{REFERENCES}

[1] J. Fu, R. B. Schoch, A. L. Stevens, S. R. Tannenbaum, and J. Han, "A patterned anisotropic nanofluidic sieving structure for continuous-flow separation of DNA and proteins," Nat. nanotechnol. vol. 2, pp. 121-128, 2007.

[2] D. Huh, K. L. Mills, X. Zhu, M. A. Burns, M. D. Thouless, and S. Takayama, "Tuneable elastomeric nanochannels for nanofluidic manipulation," Nat. Mater. vol. 6, pp. 424-428, 2007.

[3] R. Mukhopadhyay, "What Does Nanofluidics Have to Offer?," Anal. Chem. vol. 78, pp. 7379-7382, 2006.

[4] M. E. Sandison, and J. M. Copper, "Nanofabrication of electrode arrays by electron-beam and nanoimprint lithographies," Lab Chip vol. 8, pp. 
1020-1025, 2006.

[5] H. Yanagi, and Y. Kawai, "Organic Field-Effect Transistor with Narrow Channel Fabricated Using Focused Ion Beam," Jpn. J. Appl. Phys. vol. 43, pp. L1575-L1577, 2004.

[6] J. A. van Kan, A. A. Bettiol, and F. Watt, "Three-dimensional nanolithography using proton beam writing," Appl. Phys. Lett. vol. 83, pp. 1629-1631, 2003.

[7] S. A. Gajar, and M. W. Geis, "An Ionic Liquid - Channel Field - Effect Transistor," J. Electrochem. Soc. vol. 139, pp. 2833-2840, 1992.
[8] S. Y. Chou, P. R. Krauss, and P. J. Renstrom, "Imprint Lithography with 25-Nanometer Resolution," Science vol. 272, pp. 85-87, 1996.

[9] M. J. de Boer, R. W. tjerkstra, J. W. Berenschot, H. V. Jansen, G. J. Burger, J. G. E. Gardeniers, M. Elwenspoek, and A. van den Berg, "Micromachining of buried micro channels in silicon," J. Microelectromech. Syst. vol. 9, pp. 94-103, 2000.

[10] Q. He, S. Chen, Y. Su, Q. Fang, and H. Chen, "Fabrication of 1D nanofluidic channels on glass substrate by wet etching and room-temperature bonding," Anal. Chim. Acta vol. 628, pp. 1-8, 2008. 